\title{
Infection of aortobifemoral bypass graft implanted 20 years ago proved by labeled Ieukocytes SPECT-CT
}

Olgierd Chrabanski1 ${ }^{10}$, Tomasz Golab²

'Department of Radiodiagnostics, Invasive Radiology and Nuclear Medicine, Faculty of Medical Sciences in Katowice, Medical University of Silesia, Katowice, Poland

${ }^{2}$ Clinica Medica, Tychy, Poland

[Received 30 III 2021; Accepted 7 V 2021]

KEY words: infection; aortobifemoral; graft; labeled leukocytes; SPECT-CT

Nucl Med Rev 2021; 24, 2: 115-117

Aortic graft infections are very serious complications of arterial reconstructive surgery. The described in literature longest interval between primary reconstruction and aortic graft infections was 20 years [1].

This research reports a case of a 60-year-old man with a suspected infection of an aortobifemoral bypass graft implanted 20 years ago due to an aneurysm of the abdominal aorta. He was referred to a nuclear medicine department for scintigraphic detection or exclusion of active infection within the stent-graft.

The patient had many comorbidities: renal failure, arterial hypertension, hypertensive cardiomyopathy, ischemic heart disease. This year, the patient underwent thrombosis of the left saphenous vein with cellulitis of the left leg and was treated surgically due to an abscess of the left buttock. Laboratory tests revealed: leukocytosis, significantly elevated CRP and anaemia. Enterococcus faecalis susceptible to ampicillin, teicoplanin and vancomycin were grown on venous blood cultures. Despite the implementation of antibiotic therapy, no significant improvement in the patient's clinical condition was achieved. The computed tomography examination showed changes suggesting infection of the vascular prosthesis.

Due to the high risk of the surgery, it was decided to operate after obtaining scintigraphic detection of infection within the stentgraft.

The examination was performed with Technetium-99m labelled leukocytes using HMPAO.

The patient was examined 1, 4 and 24 hours after radiotracer injection using Symbia Intevo with a protocol including SPECT/CT with LEHR collimator, low-dose CT.

Correspondence to: Olgierd Chrabanski, Department of Radiodiagnostics, Invasive Radiology and Nuclear Medicine, Faculty of Medical Sciences in Katowice, Medical University of Silesia, Katowice, Poland,

e-mail: olgierdchrabanski@gmail.com

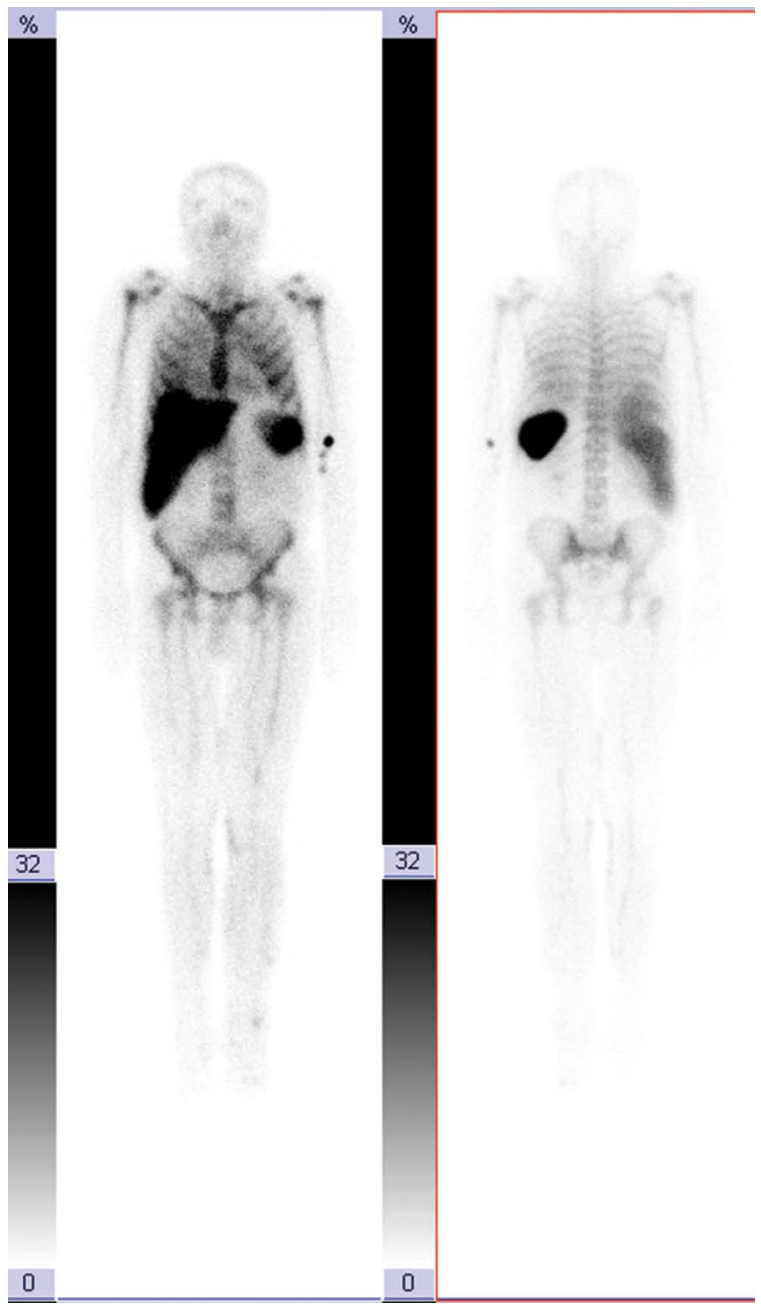

Figure 1. Whole-body WBC scan performed 4 hours after the administration of the radiotracer, no obvious foci of pathological accumulation 99mTc-HMPAO labelled leukocytes in stent-graft

This article is available in open access under Creative Common Attribution-Non-Commercial-No Derivatives 4.0 International (CC BY-NC-ND 4.0) license, allowing to download articles and share them with others as long as they credit the authors and the publisher, but without permission to change them in any way or use them commercially. 


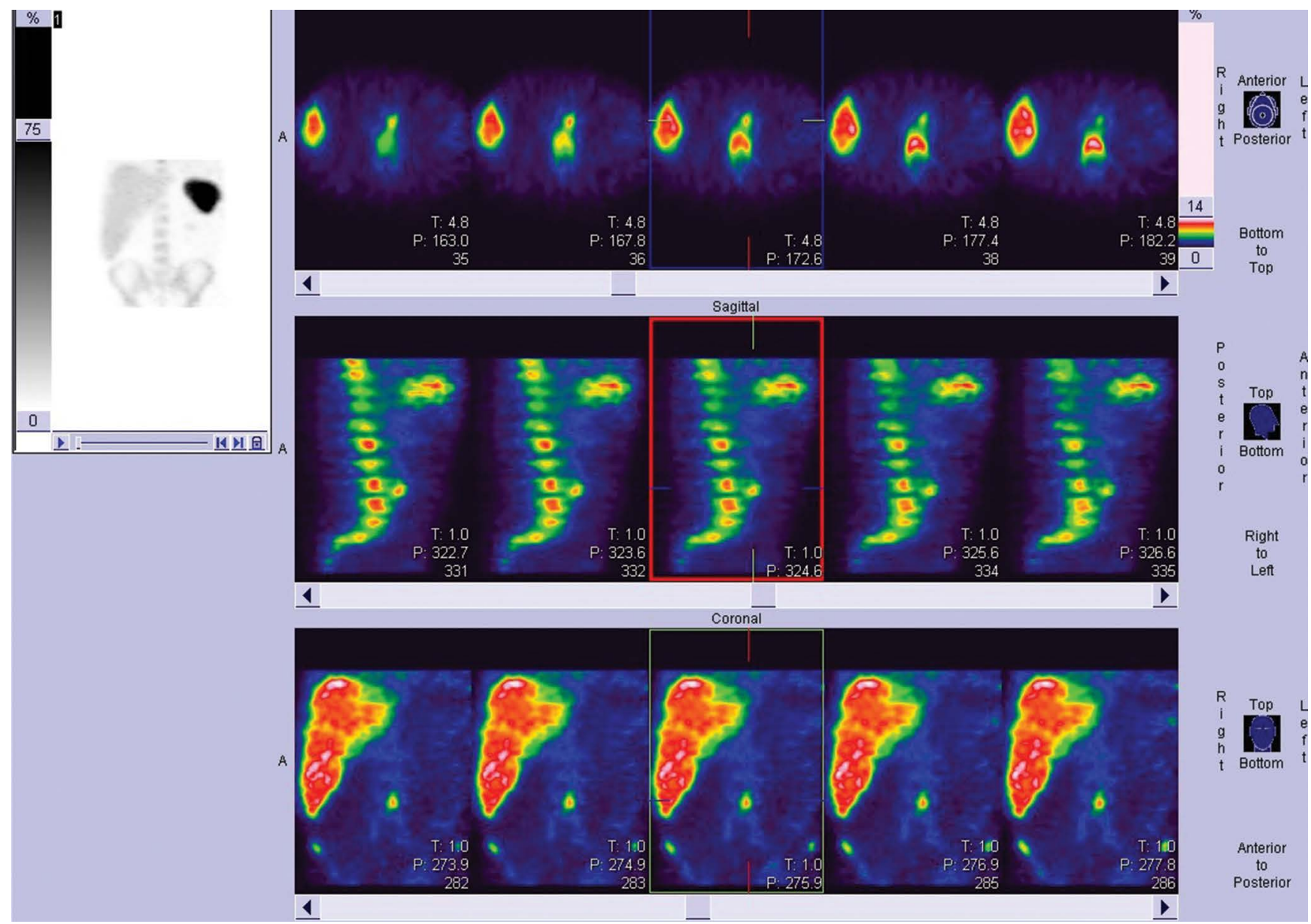

Figure 2. SPECT after 4 hours there was a small focus on the level of L4/L5 intervertebral space and slightly to the left of the midline of the body

Planar examination of the whole body performed 4 hours after the administration of the radiotracer - no obvious foci of pathological accumulation of the radiotracer in stent-graft. Discreet accumulation of labelled leukocytes was visible in the projection of the left shank, which might be a consequence of the patient's history of left saphenous vein thrombosis. The enlarged liver was also visible in Figure 1.

On SPECT after 4 hours there was a small focus on the level of L4/L5 intervertebral space and slightly to the left of the midline of the body Figure 2 .

In SPECT-CT acquisition, pathological accumulation of the radiotracer localized in the aorta (within the stent-graft) was detected in Figure $3 \mathrm{~A}-\mathrm{C}$.
After scintigraphic examination, the patient was operated on. During the operation, infection of stent-graft was confirmed. Modern gamma camera using high-resolution SPECT/CT can detect small foci invisible on the planar scan. It can be essential for final diagnosis.

\section{Conflict of interest}

The authors report no conflicts of interest.

\section{References}

1. Treska V, Certik B, Molacek J. Management of aortic graft infections - the present strategy and future perspectives. Bratisl Lek Listy. 2016; 117(3): 125-132, doi: 10.4149/bll_2016_024, indexed in Pubmed: 26925740. 

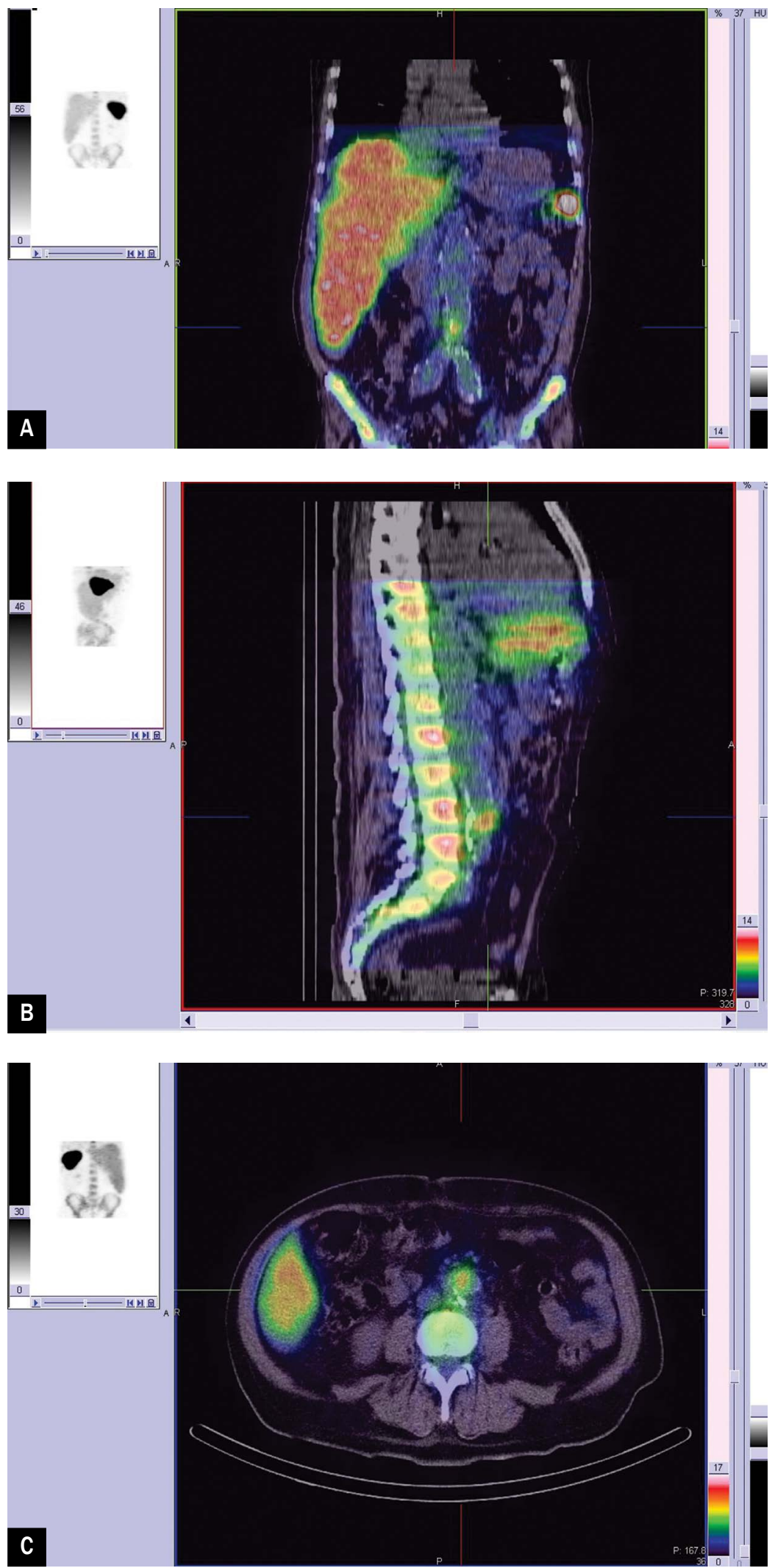

Figure 3 A-C. In SPECT-CT acquisition after $4 \mathrm{~h}$, pathological accumulation of labelled leukocytes localized in the aorta stent-graft confirm infection 